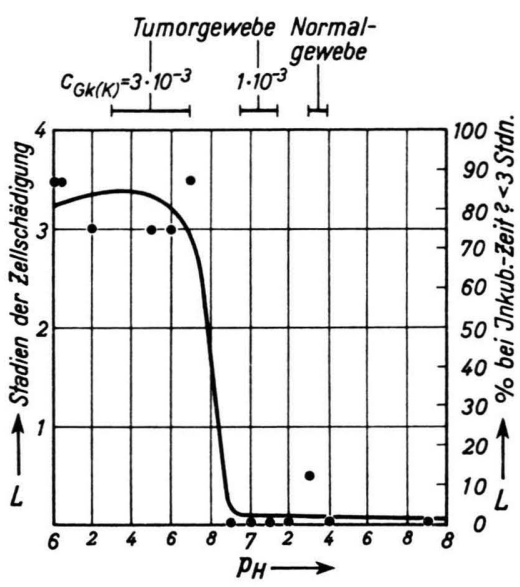

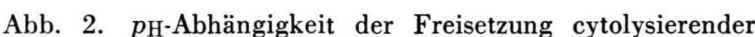
Giftstoffe aus frischen Rattenlungen-Homogenaten.
Giftstoffe aus frischen Ratten-Lungen-Homogenaten ${ }^{13}$. Ein spezieller Weg zur irreversiblen katalytischen Aufgiftung, den wir gegenwärtig erkunden, ist die $\mathrm{H}^{\circ}$-Ionen-katalysierte Abspaltung von z. B. an Peptiden gebundenen Zellgiften, wobei das Trägerpeptid nach Freigabe des Giftes eine irreversible Umlagerung erfährt.

Für die Förderung dieser Arbeit haben wir dem Ministerium für Gesundheitswesen der D D R zu danken. Für anregende Diskussionen zur Tab. 1 sind wir Herrn W. Krüger und Herrn R. TümmLER zu Dank verpflichtet.

13 H. Druckrey et al., Z. Krebsforsch. 63, 28 [1959].

\title{
Fraktionierung säurelöslicher Proteine aus Normalleber und Hepatom der Ratte *
}

\section{K. D. Schwenke ** und M. KuJawa}

Arbeitsgruppe Dr. med. habil. F. Schмid und Bereich „Chemie der Fremdstoffe in Nahrung und Ernährung“ (Leiter Dr. R. ENGST), Institut für Ernährung der Deutschen Akademie der Wissenschaften zu Berlin in Potsdam-Rehbrücke ***

(Z. Naturforschg. 22 b, 961-966 [1967] ; eingegangen am 10. Februar 1967)

Es wird die Fraktionierung der säurelöslichen Proteine aus Rattenleber- und Hepatomextrakten mit Hilfe von Stärkegel-Elektrophorese, freier Elektrophorese und Gelfiltration beschrieben. Mit Hilfe der freien Elektrophorese konnten die extrahierten Proteine den Proteinfraktionen des Gewebehomogenates zugeordnet werden. Neben den basischen h- und i-Proteinen enthalten die Extrakte Albumin und Globuline der a-, b- und g-Gruppe.

Säureextrakte aus Leber und Hepatom unterscheiden sich durch ihre Absorption im sichtbaren und ultravioletten Spektralbereich, durch ihr Elutionsprofil bei der Gelfiltration und ihr Verhalten bei der Stärkegel-Elektrophorese. Ein basisches Chromoproteid mit charakteristischer Absorption bei $400 \mathrm{~nm}$ fehlt in den Extrakten aus Hepatom.

Die Verwendung von 1,5-m. Harnstoff zur Stärkegel-Elektrophorese der salzlöslichen Organproteine führte zu einer wesentlichen Verbesserung der Proteintrennung.

Der Vergleich der elektrophoretisch aufgetrennten Proteinfraktionen aus Leber und Hepatom der Ratte ${ }^{1}$ sowie aus Niere und Nierencarcinom des Galdhamsters ${ }^{1,2}$ ergab eine Abnahme bzw. ein Fehlen gewisser basischer Proteinfraktionen (h- und i-Protein) beim Tumor. Diese quantitative oder sogar qualitative Verschiedenheit zwischen den Tumor-

* 6. Mitt. über „Proteine aus Tumor- und Normalgewebe“, 5. Mitt. s. 1. c. ${ }^{8}$.

** Neue Anschrift: Institut für Krebsforschung der DAW zu Berlin, Außenstelle Potsdam-Rehbrücke.

*** Ein wesentlicher Teil dieser Arbeit wurde in der ehemaligen Forschungsstelle für experimentelle Onkologie der Deutschen Akademie der Wissenschaften zu Berlin in Potsdam-Rehbrücke, durchgeführt.

1 K. D. Schwenke, Z. Krebsforsch. 68, 112 [1966].

2 K. D. Schwenke, R. Plass u. M. Kujawa, Z. Krebsforsch. 68, 234 [1966]. und Normalgewebsproteinen steht in Übereinstimmung mit den Arbeiten anderer Autoren, nach denen die h-Proteine des Normalgewebes Cancerogene zu binden vermögen, wogegen die entsprechenden Fraktionen des Tumorgewebes diese Fähigkeit verloren haben ${ }^{3-6}$. Basische Proteine, wie z. B. Histone, erwiesen sich darüber hinaus als Stoffe mit stark för-

3 S. Sorof u. P. P. Cohen, J. biol. Chemistry 190, 311 [1951].

${ }^{4}$ S. Sorof u. P. P. Cohen, Cancer Res. 11, 376 [1951].

5 S. Sorof, P. P. Cohen, E. C. Miller u. J. A. Miller, Cancer Res. 11, 383 [1951].

6 S. Sorof, E. M. Young u. M. G. Otт, Cancer Res. 18, 33 [1958].

7 K. D. Schwenke, Z. Chem. 7, 91 [1967].

8 K. D. Schwenke u. M. Kujawa, Z. Krebsforsch. 70, 64 [1967]. 
dernder oder hemmender Wirkung auf gewisse fundamentale Stoffwechselvorgänge ${ }^{7}$. In dieser Arbeit werden die säurelöslichen, cytoplasmatischen Gewebeextrakte aus Rattenleber und -hepatom mit Hilfe von Elektrophorese und Gelfiltration vergleichend untersucht.

\section{Experimenteller Teil}

Die Aufarbeitung der Gewebe erfolgte wie bereits beschrieben ${ }^{1,2}$. Das Homogenat wurde bei $80000 \mathrm{~g}$ zentrifugiert, zum klaren Überstand unter Rühren in kleinen Portionen das gleiche Volumen 1-n. Schwefelsäure gegeben (Endkonzentration 0,5-n.) und 1 bis 2 Stdn. bei $+4{ }^{\circ} \mathrm{C}$ gerührt. Anschließend wurde eine Stde. bei 10000 bis $15000 \mathrm{U} / \mathrm{min}$ in einer Kühlzentrifuge bei $0^{\circ}$ zentrifugiert, der klare, gelbliche (bei Hepatom-Extrakten farblose) Überstand gegen Wasser bis zum $p_{\mathrm{H}} 5$ dialysiert und mit dem dreifachen Volumen Aceton unter Kühlung gefällt. Nach Stehen bei $-10^{\circ}$ wurde abzentrifugiert und der wasserlösliche Anteil des Rückstandes (Fraktion A) aus Wasser/Aceton umgefällt (nicht löslicher Anteil: Fraktion B), mit Aceton und Äther gewaschen, und über G4-Glasfilternutschen (Schott/Jena) abgesaugt. Das Produkt (Fraktion A) aus Leber stellt ein graues Pulver dar, das sich leicht mit schwach gelber Farbe in Wasser löst. Ausbeute: $100-150 \mathrm{mg}$ aus $100 \mathrm{~g}$ gewaschener Leber. Das Produkt aus Hepatom, ein farbloses Pulver, löst

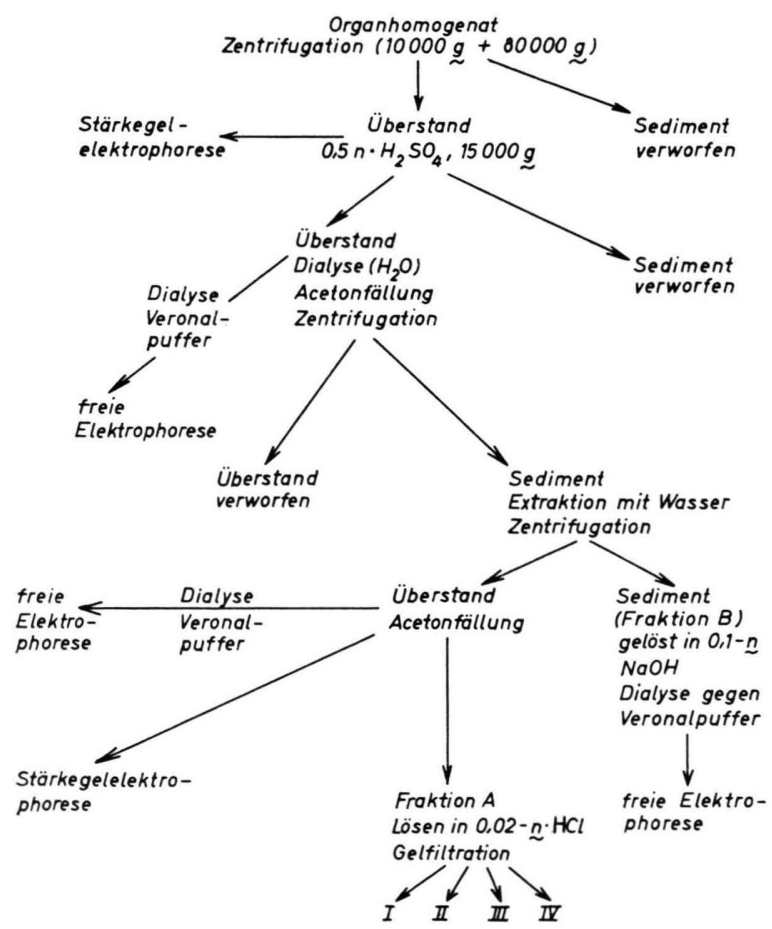

Abb. 1. Schema der Fraktionierung der Organproteine. sich klar und farblos in Wasser auf. Ausbeute: $150 \mathrm{mg}$ aus $100 \mathrm{~g}$ Hepatomgewebe. Die Produkte sind frei von Phosphor. Abb. 1 gibt die in der vorliegenden Arbeit beschrittenen Wege der Fraktionierung wieder.

Die freie Elektrophorese wurde wie bereits beschrieben ${ }^{1}$ durchgeführt. Nach Dialyse des Überstandes aus der Säureextraktion oder der wäßrigen Lösung der Fraktion A gegen den Elektrophoresepuffer (M i chaelis-Veronalpuffer nach Wiedemann, $p_{\text {H }}$ 8,55 , Ionenstärke 0,1 ) wurde der Niederschlag abzentrifugiert und die Überstände zur Elektrophorese eingesetzt $(0,5$ bis $1 \%$ Eiweiß) .

Die Elektrophorese im Stärkegel erfolgte, wie von uns an anderer Stelle beschrieben, in Boratpuffer ${ }^{8}$. Zur besseren Auftrennung der Proteine wurde dem Gel nach RösLER ${ }^{9} 1,5-m$. Harnstoff zugesetzt.

Die Gelfiltration an Sephadex wurde mit G-75- und G-200-Gelen in Säulen mit den Dimensionen $38 \cdot 0,9$ bzw. $100 \cdot 0,9 \mathrm{~cm}$ durchgeführt. Die Gele wurden durch Quellen in Wasser und Äquilibrieren mit $0,02-n$. HCl bereitet. $10 \mathrm{mg}$ der Fraktion A wurden in $0,3-0,5 \mathrm{ml}$ 0,02-n. HCl gelöst und mittels einer Glaspräzisionsspritze bei dem G-75-Gel auf den Kopf der Säule aufgetragen; bei G-200-Säulen wurde das Elutionsmittel oberhalb des Gels mit der Probe unterschichtet. Bei einer Tropfgeschwindigkeit von 1 Tropfen/20 $-30 \mathrm{sec}$ (ca. $15 \mathrm{ml} / \mathrm{Stde}$.) wurde mit $0,02-n . \mathrm{HCl}$ eluiert und Fraktionen von $1,2-2 \mathrm{ml}$ mittels eines automatischen Fraktionssammlers aufgefangen. Die Eiweißkonzentration in den Fraktionen wurde bei $278 \mathrm{~nm}$ mit dem Universalspektrophotometer VSU1 (VEB Zeiss Jena) gemessen. Vergleichsweise wurden Messungen der Absorption im ultravioletten und sichtbaren Spektralbereich für die Originalsubstanzen und die nach der Gelfiltration erhaltenen Fraktionen mit dem selbstregistrierenden Spektralphotometer USP2 (Werkstätten der DAW zu Berlin, Berlin-Adlershof) durchgeführt.

Für die Stärkegel-Elektrophorese wurden die Filtrate der Gelfiltration durch Dialyse gegen eine wäßrige Lösung (1:1) von Carbowax 6000 konzentriert. Dazu dienten die mit einer dünnen Cellophanfolie $(20 \mu$ stark) bespannten, vom VEB Zeiss Jena für die Elektrophorese-Apparaturen gelieferten Dialysatoren. $10 \mathrm{ml}$ Filtrat wurden so bei Zimmertemperatur innerhalb von $5 \mathrm{Stdn}$. auf $0,5 \mathrm{ml}$ eingeengt. Zur Entfernung geringer Mengen Carbowax im Dialysat wurde anschließend gegen Wasser dialysiert und mit dem dreifachen Volumen Aceton gefällt. Nach 2-stdg. Stehen bei $-10^{\circ} \mathrm{C}$ wurde abzentrifugiert und das Sediment in wenig $(0,2$ bis $0,3 \mathrm{ml})$ Wasser gelöst.

Die Eiweißbestimmungen in den Gewebeextrakten wurden mit dem Folin-Reagenz nach Lowry und Mitarb. ${ }^{10}$ durchgeführt.

9 B. Rösler, Acta biol. med. German. 16, 106 [1966].

10 O. H. Lowry, N. J. Rosebrovgh, A. L. Farr u. R. J. Randall, J. biol. Chemistry 193, 265 [1951]. 


\section{Ergebnisse}

Die Fraktionierung der Gesamtextrakte mit Hilfe der Stärkegel-Elektrophorese

Die Auftrennung der Originalextrakte aus Leber und Hepatom mit Hilfe der Stärkegel-Elektrophorese lieferte zahlreiche mehr oder weniger diffuse Banden. Der Einsatz von Harnstoff verbesserte die Trennung außerordentlich. Die Abbn. $2 \mathrm{a}$ und $2 \mathrm{~b}$ zeigen

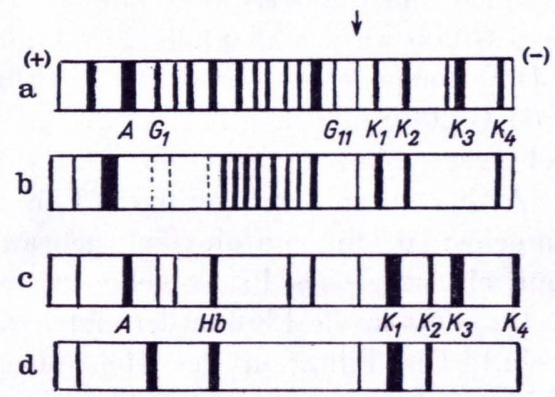

Abb. 2. Schematische Wiedergabe von Elektrophoresebildern im 1,5-m. Harnstoff-Stärkegel; $\downarrow$ Auftragstelle. a) Leber-Originalextrakt, b) Hepatom-Originalextrakt, c) Leber-Säureextrakt, Fraktion A, d) Hepatom-Säureextrakt, Fraktion A.

die Elektropherogramme der Leber-(a) - und Hepatom-(b)-Homogenate nach Auftrennung in einem Gel mit 1,5-m. Harnstoff. Beide sind deutlich voneinander unterschieden. Der Leberextrakt weist insgesamt 20 scharfe Banden auf. Neben je einer Albumin- und zwei Präalbuminbanden sind 11 GlobulinZonen $\left(G_{1}-G_{11}\right)$ auf der Anodenseite sichtbar. Kathodisch laufen 4 Proteinbanden $\left(\mathrm{K}_{1}, \mathrm{~K}_{2}, \mathrm{~K}_{3}\right.$, $\mathrm{K}_{4}$ ), von denen $\mathrm{K}_{3}$ eine Doppelzone ausbildet. Die $\mathrm{K}_{3}$-Doppelbande fehlt im Hepatomextrakt. Ebenso fehlen im Bereich der $G_{1}$ - bis $G_{11}$-Globuline einige im Leberextrakt deutlich sichtbare Banden (Abb. 2 a und $\mathrm{b})$. Hingegen sind einige Globulinzonen im Hepatomextrakt im Bereich der $\mathrm{G}_{4}$ - bis $\mathrm{G}_{10}$-Fraktionen zu sehen, die im Leberextrakt fehlen. Aus unseren Erfahrungen mit Extrakten aus GoldhamsterNiere ${ }^{2}$ bzw. den Angaben der Literatur ${ }^{11}$ schließen wir, da $ß$ die von uns nachgewiesenen Proteinzonen keine einheitlichen Proteine darstellen, sondern jeweils aus einer größeren Zahl von Komponenten bestehen.

11 B. W. Moore, P. U. Angeletti, V. Suntzeff, G. Gayle u. R. Davenport, Acta Univ. Cancrum 20, 947 [1964].

12 D. I. Lloyd u. A. Shore, „Chemistry of Proteins“, London 1938, S. 291.

\section{Fraktionierung der Säureextrakte mit Hilfe der freien Elektrophorese}

Die Fraktionen A aus Leber und Hepatom lassen sich nach Elektrophorese in $\mathrm{Mich}$ a elis-Puffer in zahlreiche Subfraktionen auftrennen. Wie aus der Säurelöslichkeit der Albumine und Globuline ${ }^{12-14}$ zu erwarten war, enthalten die Säureextrakte Albumin- und Präalbumin-Fraktionen, ferner a-, b- und g-Fraktionen. Die h-Protein-Fraktionen und die basischen i-Fraktionen der Leber sind jedoch gegenüber dem Originalhomogenat eindeutig angereichert. Abb. 3 zeigt die Elektrophoresekurven der Säureextrakte (A-Fraktionen) aus Leber (3 a) und Hepa-

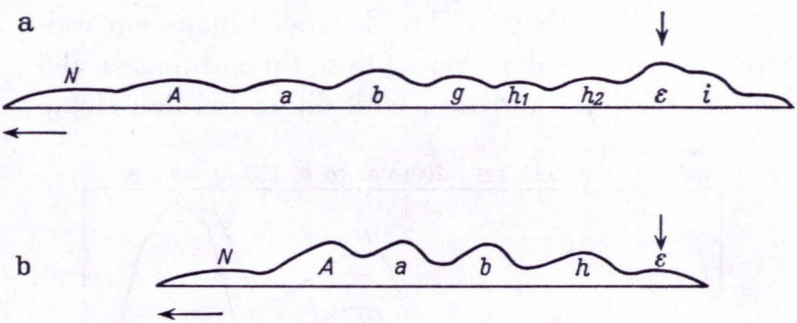

Abb. 3. Auftrennung der A-Fraktionen aus Leber (a) und Hepatom (b) mit Hilfe der freien Elektrophorese $\leftarrow$ anodische Wanderung, $\downarrow$ Nullstellung.

tom $(3 \mathrm{~b})$. Die Zuordnung der Fraktionen erfolgte auf Grund ihrer elektrophoretischen Beweglichkeiten. Die Bezeichnung entspricht derjenigen in unserer ersten Mitteilung ${ }^{1}$. Aus der Zahl der Fraktionen, ihrer relativ scharfen Abtrennung und ihrer Ausbildung in relativ symmetrischen Gipfeln läßt sich schließen, daß die überwiegende Zahl der Fraktionen des Originalextraktes durch die Säurebehandlung denaturiert wurde und nur eine verhältnismäßig geringe Anzahl von Proteinfraktionen im Extrakt vorliegt. Leberextrakte enthalten neben stark ausgebildeten h-Fraktionen zwei gut sichtbare, kathodisch wandernde (i-) Fraktionen (Abb. 3 a). Insgesamt lassen sich $8 \mathrm{Gipfel}$ eindeutig erkennen. Die Extrakte aus Hepatom ergeben mit 5 scharf ausgebildeten Gipfeln ein von dem der Leber abweichendes Bild (Abb. 3 b). Die Gipfel entsprechen einem Präalbumin (N) sowie den A-, a-, b- und h-Fraktionen; die h-Fraktion $\left(h_{1}\right)$ wurde stark angereichert.

13 F. Samuely u. E. Strauss, „Eigentliche Proteine“, in: Handbuch der biol. Arbeitsmethoden, Abt. I, Chem. Meth. Teil 8, S. 461. Herausgeber E. Abderhalden, Berlin-Wien 1922.

14 M. SpIegel-Adolf, „Die Globuline“, in: Handbuch der Kolloidwissensch. Dresden u. Leipzig 1930, Band IV, S. 189. 
Unter gleichen Bedingungen ergab die B-Fraktion nach Auflösung in 0,1-n. $\mathrm{NaOH}$ und Dialyse gegen $\mathrm{M}$ i c h a el is - Puffer nur einen Gipfel geringer Beweglichkeit (etwa der g-Fraktion entsprechend); offenbar handelt es sich dabei um durch Acetonfällung denaturierte Proteine.

\section{Spektren der Säureextrakte}

Abb. 4 zeigt die Absorption der Leber (L) - und Hepatom (H)-Säureextrakte (Fraktionen A) im ultravioletten und sichtbaren Spektralbereich. Im Bereich der typischen Eiweißabsorption bei $278 \mathrm{~nm}$ erkennt man ein Maximum, das bei der Hepatomkurve nach dem längerwelligen Bereich verschoben ist. Während die Leberextrakte darüber hinaus ein weiteres Maximum der Absorption im sichtbaren Bereich bei $398 \mathrm{~nm}$ aufwies, fehlt dieses bei den Hepa-

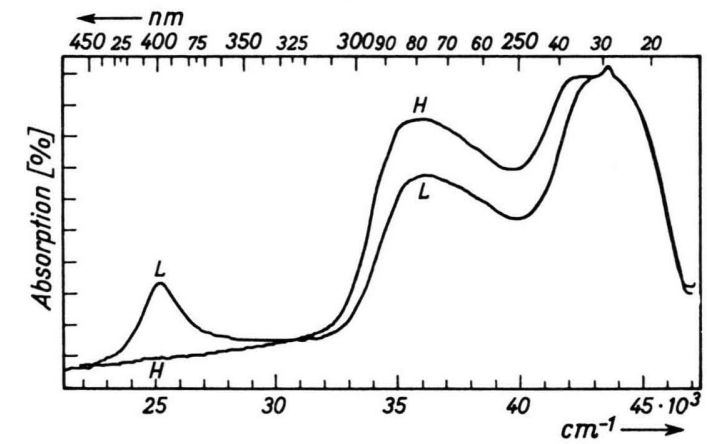

Abb. 4. Absorptionsspektren der Leber (L) - und Hepatom (H) . A-Fraktion.

tomextrakten. Die Absorption bei $398 \mathrm{~nm}$ ist also offenbar auf Chromophore zurückzuführen, die an Proteinzonen der Leber gebunden sind, welche im Hepatom fehlen. Die trotz gleicher Substanzkonzentration $(1 \mathrm{mg} / \mathrm{ml})$ des Leber- und Hepatomextraktes größere Absorption der Hepatom-Proteine bei $278 \mathrm{~nm}(85 \%$, Leber 67\%) könnte ihre Ursache in einer Verminderung der Proteinkonzentration des Leberextraktes zugunsten einer Protein-gebundenen chromophoren (Absorption bei $398 \mathrm{~nm}$ ) Nichtproteinkomponente oder aber in einem höheren Anteil von bei $278 \mathrm{~nm}$ absorbierenden chromophoren Bestandteilen (z. B. Tyrosin) der Hepatomproteine haben. Die Eiweißbestimmung nach Lowry et al. ergab für die Lösung des Leberextraktes beim Vergleich mit Rinderserumalbumin den der Einwaage entsprechenden Gehalt von $1 \mathrm{mg} / \mathrm{ml}$. Dagegen wurde für den Hepatomextrakt eine höhere Extinktion entsprechend einem Gehalt von 1,3 mg Eiweiß/ml bei gleicher Einwaage gefunden.

\section{Gelfiltration der Säureextrakte}

Die Gelfiltration der Leberextrakte (Fraktion A) an Sephadex G-75 und G-200 ergab jeweils drei scharfe Gipfel (Messung der UV-Absorption) (Fraktionen $\mathrm{I}_{\mathrm{L}}, \mathrm{III}_{\mathrm{L}}, \mathrm{IV}_{\mathrm{L}}$ ), von denen der mittlere (Fraktion III) die bei $398 \mathrm{~nm}$ absorbierende Komponente enthält (Abb. 5). Die zwischen den Fraktionen $I_{L}$ und $\mathrm{III}_{\mathrm{L}}$ liegende Schulter wurde als Fraktion $\mathrm{II}_{\mathrm{L}}$ entnommen. Die Eluogramme der Hepatom-A-Fraktionen zeigten einen anderen Verlauf. Nach Filtration durch G-200 wurden ebenfalls 4 Fraktionen erhalten. Die Komponente I erscheint im Eluat der G-75- und G-200-Säule in den ersten Röhrchen als vom Gel ausgeschlossene Fraktion. (Da die Substanzen A in einem sehr geringen Flüssigkeitsvolumen gelöst $[0,3 \mathrm{ml}]$ auf die Säule gebracht wurden, kann ein zu großes Probenvolumen nicht die Ursache für die schnelle Elution der ersten Komponente sein.) Die Filtration der Hepatomextrakte durch G-75 ergab eine weitaus schlechtere Trennung. Abb. 5 zeigt das Eluogramm der A-Fraktionen aus

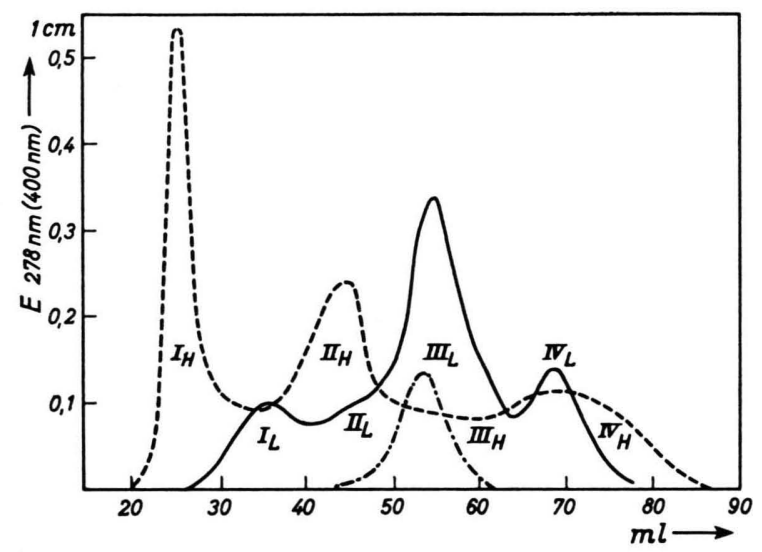

Abb. 5. Gelfiltration der Leber- und Hepatom-(A)-Fraktionen durch Sephadex-G-200 $(95 \times 0,9 \mathrm{~cm})$. Leber bei $278 \mathrm{~nm}$, - - - - Leber bei $400 \mathrm{~nm},---$ Hepatom bei $278 \mathrm{~nm}$.

Leber und Hepatom nach Benutzung einer G-200Säule. Das sehr unterschiedliche Elutionsprofil und die Lage der Gipfel bei den Leber- und Hepatomproteinen deuten auf charakteristische Unterschiede zwischen beiden Geweben bezüglich der Zusammensetzung der ersten Fraktionen (I und II) beider Gewebe hin.

\section{Stärkegel-Elektrophorese der Säureextrakte und Fraktionen der Gelfiltration}

Wie die Zonenelektrophorese der A-Fraktionen im Harnstoff-Stärkegel ergab (Abbn. $2 \mathrm{c}$ und d), wur- 
den durch die Säurebehandlung der Leberhomogenate 12 Proteinfraktionen extrahiert. Aus den Proteinzonen des Leber-Säure-Extraktes heben sich besonders zwei anodisch wandernde, dem Albumin und dem Hämoglobin entsprechende, und vier kathodisch wandernde Zonen (K-Fraktionen) ab. Die übrigen 6 Banden sind sehr schwach und liegen im Bereich der Präalbumine, Postalbumine $\left(G_{1}, G_{2}\right)$ bzw. der langsam wandernden $\alpha_{1}$ - und $\alpha_{2}$-Globuline $\left(G_{8}\right.$ bis $\mathrm{G}_{\mathbf{1 0}}$ ). Hepatom-Säureextrakte ergaben Elektropherogramme mit nur 7 Fraktionen. Davon sind 4 (A, $\mathrm{Hb}, \mathrm{K}_{1}, \mathrm{~K}_{2}$ ) (Abb. $2 \mathrm{~d}$ ) sehr stark ausgebildet. Die in den Elektropherogrammen der Homogenate gefundenen Unterschiede zwischen beiden Geweben treten hier noch deutlicher zutage.

Die Elektropherogramme der Fraktionen I-IV des Leberextraktes zeigen, daß eine echte Fraktionierung stattgefunden hat (Abbn. $6 \mathrm{a}-\mathrm{f})$. Fraktion I enthält nur die schnell laufenden sauren Proteine (Albumin, Hb, 2 Globuline). Fraktionen II und III bestehen aus 3 starken kathodischen Banden sowie einigen schwächeren anodisch wandernden Zonen.

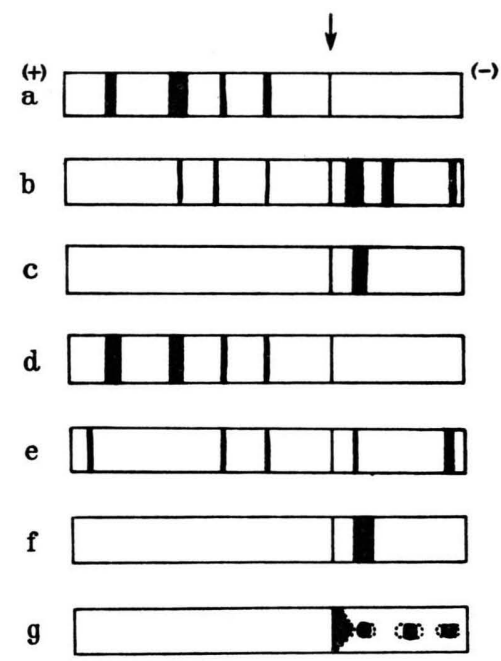

Abb. 6. Schematische Wiedergabe von Elektrophoresebildern der Hauptfraktionen der Gelfiltration; $\downarrow$ Auftragstelle. a) Leber, Fraktion $\mathrm{I}_{\mathrm{L}}$, b) Leber, Fraktion $\mathrm{III}_{\mathrm{L}}$, c) Leber, Fraktion $\mathrm{IV}_{\mathrm{L}}$, d) Hepatom, Fraktion $\mathrm{II}_{\mathrm{H}}$, e) Hepatom, Fraktion $\mathrm{III}_{\mathrm{H}}$,

f) Hepatom, Fraktion IVH, g) Hepatom, Gesamthistone.

Fraktion IV schließlich enthält nur eine starke kathodische Bande. Fraktion I und IV sind frei von den im Sichtbaren absorbierenden chromophoren Komponenten. Diese befinden sich in den Banden der Fraktion III. Im Elektropherogramm der Fraktion III aus Hepatom-Säureextrakten fehlen die star- ken kathodischen Banden. Diese enthalten demnach (Vergleich der Absorptionsspektren der Leber- und Hepatomfraktionen) die stark chromophore Komponente an ein Protein gebunden. Fraktion I und II unterscheiden sich elektrophoretisch kaum; sie enthalten ausschließlich anodisch laufende Proteine. Als letzte Fraktion erscheint im Gelfiltrat sowohl bei Leber- als auch bei Hepatomextrakten ein kathodisch laufendes Protein. Auf Grund des Verhaltens bei der Gelfiltration (Fraktion mit dem niedrigsten Mol.Gew.) und der elektrophoretischen Wanderung könnte es sich dabei um ein histonartiges Protein handeln ${ }^{7}$. Eine Entscheidung darüber muß weiteren Versuchen, die eine Aminosäuren-Analyse einschließen, vorbehalten bleiben. In Abb. $6 \mathrm{~g}$ ist das Elektropherogramm der Gesamthistone aus Hepatom wiedergegeben. Wie aus der Aggregationstendenz einiger Histonfraktionen bei $p_{\mathrm{H}}$-Werten $>5$ zu erwarten ist $^{7}$, verbleibt die Hauptmenge der Proteine am Start. Einige Fraktionen wandern aber kathodisch. Es könnte sich hierbei um die lysinreichen Fraktionen handeln, die nicht aggregieren.

\section{Diskussion}

Stärkegel-Elektrophorese, Gelfiltration an Sephadex und optische Absorption der säurelöslichen Cytoplasmaproteine ergaben charakteristische Unterschiede zwischen Normalleber und Hepatom. Die vorliegenden Ergebnisse gehen über früher mit elektrophoretischen Methoden gemachte Feststellungen ${ }^{1,2}$ hinaus. Die Unterschiede zwischen den Proteinen beider Gewebe betreffen vorwiegend die basischen, bei der Elektrophorese im Stärkegel kathodisch laufenden Proteine. Auf Grund der fehlenden Absorption der kathodischen Hepatomproteine im sichtbaren Spektralbereich und einer entsprechenden Lücke im Elektropherogramm schließen wir auf das Fehlen eines basischen Chromoproteids ( $\mathrm{K}_{3}$-Protein) beim Hepatom. (Dieser Befund stimmt mit dem makroskopischen Bild überein; blutfreies Hepatomgewebe ist fast weiß, gewaschenes Lebergewebe strohgelb.) Dieses Protein ist möglicherweise identisch mit der von Burston und Mitarb. ${ }^{15}$ beschriebenen, Perchlorsäure-löslichen A-Fraktion, die als farbige Proteinkomponente in Extrakten aus menschlicher Leber nachgewiesen wurde. In menschlichen Lebertumoren war sie stark vermindert. Auf Grund ihrer elektro-

15 D. Burston, M. A. Tombs, M. E. Apsey u. N. F. Maclagan, Brit. J. Cancer 17, 162 [1963]. 
phoretischen Beweglichkeit könnte die $\mathrm{K}_{3}$-Fraktion den h-Proteinen ${ }^{3,4}$ zugeordnet werden, deren Rolle bei der Bindung von Cancerogenen im Cytoplasma von Sorof und Mitarb. ${ }^{4-6,16}$ sowie von HeIdelBERGER und Mitarb. ${ }^{17}$ untersucht wurde. Auf Grund der sehr verschiedenen Elutionskurven der Leberund Hepatomextrakte bei der Gelfiltration und der unterschiedlichen UV-Absorption der vergleichenden Fraktionen lassen sich charakteristische Unterschiede zwischen Leber- und Hepatomproteinen feststellen, die bei der Stärkegel-Elektrophorese dieser Fraktionen verborgen bleiben. Diese Unterschiede betreffen besonders die von kathodischen Proteinen freien Fraktionen mit schnell wandernden sauren Proteinen.

Die Beeinflussung wichtiger biosynthetischer Reaktionen im Cytoplasma bzw. cytoplasmatischen Partikeln durch Histone ${ }^{18,19}$ läßt an eine Regulation gewisser cytoplasmatischer Stoffwechselwege durch diese Proteine denken. Da die lysinreichen Histonfraktionen offenbar an der Oberfläche der DNS im Zellkern gebunden sind und am leichtesten abdissoziieren ${ }^{7}$, könnten diese Fraktionen aus dem Zellkern in das Zellplasma wandern und dort als Regulatoren

16 S. Sorof, E. M. Young, M. M. McCue u. P. L. Fettermann, Cancer Res. 23, 864 [1963].

17 C. Heidelberger, J. cellular comparat. Physiol. 64, 129 [1964]. fungieren. Die Isolierung einer basischen Proteinfraktion mit relativ niedrigem Mol.-Gew. (letzte Fraktion im Eluat der Gelfiltration) und einer definierten elektrophoretischen Wanderung bei $p_{\mathrm{H}} 8,6$ deutet auf Proteine mit ähnlichen Eigenschaften hin wie die lysinreichen Histone. Wenn man von einer Wechselwirkung mit anderen Proteinen des Organextraktes, die die elektrophoretische Beweglichkeit der Histone verändern kann, absieht, könnten die in der freien Elektrophorese bei $p_{\mathrm{H}} 8,6$ kathodisch wandernden i-Fraktionen histonartigen Proteinen (isoelektrischer Punkt der Histone 10 - 11) entsprechen. Allerdings können wir auf Grund unserer Gewebeaufarbeitung eine teilweise Zerstörung von Zellkernen, wodurch geringe Mengen von Histonen in den Extrakt gelangen können, nicht mit Sicherheit ausschließen. Weitere Versuche in Richtung auf eine Gewinnung definierter basischer Proteinfraktionen aus dem Cytoplasma sowie ihre chemische Charakterisierung sind im Gange.

Für gewissenhafte technische Mitarbeit haben wir Frau H. Beyer zu danken.

18 A. Schwartz, J. biol. Chemistry 240, 939, 944 [1965].

19 B. S. McEwen, V. G. Allfrey u. A. E. Mirsky, J. biol. Chemistry 238, 758 [1963]. 